\title{
Optimization method of electric field inverse problem based on intelligent algorithm
}

\author{
Qiang Zhao*, Shenglan Yang, Li Lu, Zefeng Jiang, Wei Yang, and Zhipeng Huang \\ State Grid Sichuan of Electric Power Maintenance Company, 610000 Chengdu Sichuan, China
}

\begin{abstract}
With the expansion of China's power grid, the scale, transmission distance and load capacity of transmission projects are all growing rapidly. Meanwhile, the problem of transmission line running state detection is also attracting more and more attention. Electric field under high voltage transmission line is taken as the research object in this paper, and the principle of electric field inverse operation is analyzed, two kinds of calculation objects and methods of nondestructive examination are are described in detail. There are two kinds of intelligent algorithms in this paper in order to solve the problem of several unknown solutions in inverse electric field operation. Correspondingly, the fitness function based on voltage value and result value is established, and the inverse electric field operation algorithm with global optimization function is proposed. A calculation example of high-voltage transmission line is also used to verify the inverse problem optimization algorithm. It is respectively proved by calculation results that the effectiveness and accuracy of the optimization method based on the two kinds of intelligent algorithms.
\end{abstract}

\section{Introduction}

Transmission line is a key part in power grid engineering, which is related to the safe and stable operation of power grid. Therefore, the detection of transmission wire is essential. At present, the status detection of transmission wires in smart grid mainly includes operating environment, wire sag, line voltage and so on ${ }^{[1]}$. The common detection method is to install the voltage transformer at the end of transmission lines. However, there are still many difficulties in the installation of voltage transformers on existing lines due to large volume of equipment, complex installation and strict insulation requirements ${ }^{[2-3]}$.

The inverse operation of electromagnetic field has been widely used in medical imaging, geological exploration and environmental exploration abroad ${ }^{[4-6]}$. More and more researches on the high voltage transmission facilities and surrounding electromagnetic fields are carried out around the country ${ }^{[7-8]}$. The Chongqing University team proposed to use Tikhonov regularization to solve the ill-conditioned problem of inverse electric field operation $^{[9-10]}$. According to the characteristics of the magnetic field, a new method of detecting the current of three-phase transmission lines by using the inverse calculation of power frequency magnetic field is proposed ${ }^{[11]}$. The team from Zhejiang University introduced the tabu search algorithm to solve the electromagnetic field inverse problem of

* Corresponding author: 985211936@qq.com 
the objective function of multiple extreme value points ${ }^{[12]}$. However, the global optimal solution of the inverse electric field problem under transmission line has not been involved in these studies.

Firstly, according to the characteristics of the electric field under the transmission line, the first chapter analyzes the electric field under the transmission line based on charge simulating method(CSM), and deduces the calculation method and application method of the inverse electric field operation. Secondly, in order to solve the problem of inverse electric field operation, inverse electric field algorithms with global optimization function is proposed, which combines CSM and two kinds of intelligent algorithms respectively. The second chapter introduces the calculation principle, steps and corresponding parameters of the algorithm. In the chapter 4 , the validity and accuracy of the two algorithms are verified by experiments, and the computational efficiency of the two algorithms is compared. In the chapter 5, the potential development and shortcomings of the research methods are summarized.

\section{Inverse problem of electric field under transmission line}

\subsection{Charge simulating method}

The power frequency electric field generated by the $50 \mathrm{~Hz}$ alternating current under the transmission line can be regarded as the static electric field whose source (charge) does not change with time. Through of Maxwell equations, the electrostatic field can be described by the following equation:

$$
\left\{\begin{array}{l}
\nabla \times E=0 \\
\nabla \cdot D=\rho
\end{array}\right.
$$

Through of the uniqueness theorem of electrostatic field, the solution of potential differential equation satisfying the given boundary condition is unique in electrostatic field. If a number of discrete charges are set outside the field to be solved, these discrete charges can solve the whole field as long as the given boundary conditions remain unchanged.

The charge simulating method is to replace the unknown continuous charge by setting discrete charge in the computation field without knowing the charge distribution. The equations of simulating charge are expressed as follows:

$$
[P] \cdot[Q]=[\varphi]
$$

Where $[P]$ is the coefficient matrix , $[Q]$ is the simulated charge matrix, and $[J$ is the potential matrix of matching points.

After solving the simulating charge $[Q]$, according to the electric field superposition theorem, the electric field intensity at a certain point is:

$$
[f] \cdot[Q]=E
$$

Where $[f]$ is the electric field coefficient matrix, $E$ is the electric field intensity at a certain point.

The commonly used simulating charge types are point charge, line charge and ring line charge, etc. This section mainly introduces the CSM of line charge. 


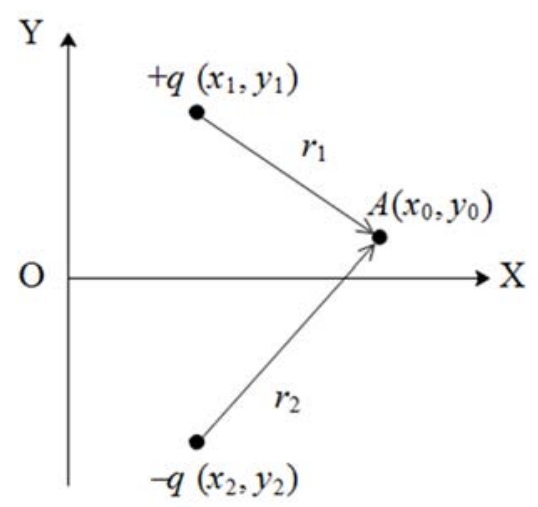

Fig.1. Symmetrical infinitely long linear charges.

When the transmission line is regarded as an infinite long line charge $+q$, a symmetrical $-q$ line charge is set with the $\mathrm{X}$-axis as the axis of symmetry based on the mirror image principle. The $\mathrm{X}$-axis is set as the potential reference line. And the potential coefficient and electric field coefficient generated at any point $A$ in the field field of the pair line charge $\operatorname{are}^{[13]}$ :

$$
\left\{\begin{array}{l}
P=\frac{1}{2 \pi \varepsilon} \ln \frac{r_{2}}{r_{1}} \\
f=\frac{1}{2 \pi \varepsilon}\left(\frac{y_{0}-y_{1}}{r_{1}^{2}}-\frac{y_{0}-y_{2}}{r_{2}^{2}}\right)
\end{array}\right.
$$

\subsection{Inverse problem of electric field under transmission line}

The relationship between the voltage on the transmission line and the offline electric field can be written as follows according to formula (2) and formula (3) :

$$
[E]=[f] \cdot[Q]=[f] \cdot\left([P]^{-1} \cdot[\varphi]\right)
$$

When the electric field intensity $[E]$ is given, the calculation of inverse operation can be applied in two aspects. On the one hand, electric field intensity [E] and charge simulation location information are given, the transmission line voltage $[\varphi]$ can be solved through the inverse calculation of coefficient matrix. On the other hand, electric field intensity $[E]$ and transmission line voltage $[\varphi]$ are given, the charge simulation location information can be solved, which is the height of the transmission wire generally.

In practical engineering application, the working flow of the voltage on the line through inverse calculation is shown in Figure 2 below. 


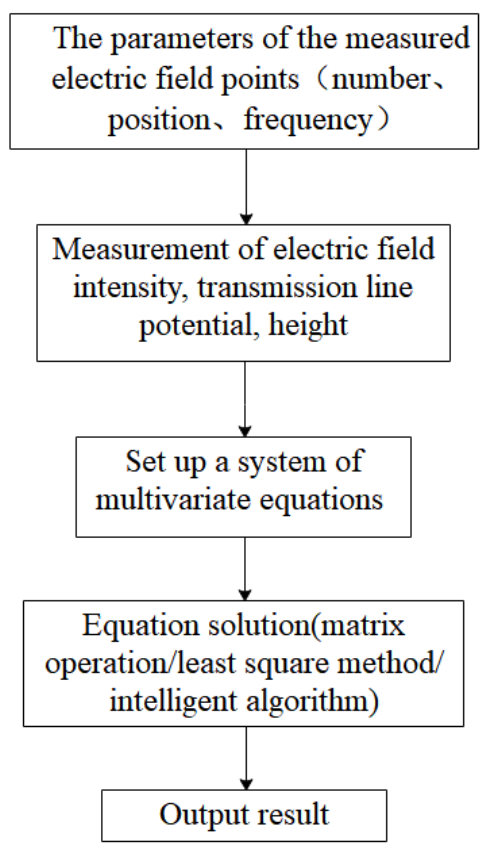

Fig. 2. The flow of voltage inverse operation on nondestructive detection.

There are two main problems to be explained in the process of solving the inverse problem. First, the calculation result, electric field value, is a scalar value synthesized by the electric field vector in three-dimensional space. For the convenience of calculation, the direction of the maximum electric field intensity $\left[E_{y}\right]$ is equal to $[E]$. Secondly, it is necessary to use mathematical methods tos olve multiple linear regression equations. The electric field intensity matrix $[E]$, coefficient matrix $[f]$ and simulated charge matrix $[Q]$ can be written as a set of multivariate linear equations. When the number of transmission line is $n$ and the number of electric field intensity measurement points is $T(T>n)$, the equation is similar to formula (6). The formula (9) is an overdetermined system composed of $T$ equations and $n$ unknowns, which means lacks exact solutions. Therefore, the solution of the multivariate linear fitting is converted to the least square method.

$$
\left[\begin{array}{l}
Q_{1} \\
Q_{2} \\
\vdots \\
Q_{n}
\end{array}\right]=\left[\begin{array}{cccc}
f_{1,1} & f_{1,2} & \cdots & f_{1, T} \\
f_{2,1} & f_{2,2} & \cdots & f_{2, T} \\
\vdots & \vdots & \cdots & \vdots \\
f_{n, 1} & f_{n, 2} & \cdots & f_{n, T}
\end{array}\right]^{-1}\left[\begin{array}{c}
E_{1} \\
E_{2} \\
\vdots \\
E_{T}
\end{array}\right]
$$

The position information of the transmission line can be solved by inverse electric field operation, namely, the height of the line. The solution process is similar to that shown in Fig. 2. Significantly, each coefficient in the coefficient matrix $[f]$ contains unknown parameters, so it is an underdetermined system composed of $T$ equations and $n \times T$ unknowns. The underdetermined equation has innumerable solutions, so it needs to be solved by using global optimization algorithm. The intelligent algorithm is used to solve the above problem in this paper. 


\section{Optimization method of electric field inverse problem based on intelligent algorithm}

\subsection{Intelligence algorithm}

Intelligent algorithm is developed from the traditional optimization algorithm, which can solve multi-objective nonlinear problems and have strong global optimization computing power. The objective function and the constraint function need not be analytic, which means well capability on all kinds of complex optimization problems.

The characteristics of solving electric field inverse problem are as follows:(1) It contains a large number of sparse coefficient matrices; (2) The search accuracy of solving parameters is in great demand; (3) Determining the optimal solution by constructing the fitness function value. Two kinds of intelligent algorithms that meet the above requirements are selected, which are combined with the CSM to construct the optimal charge simulating method(OCSM) respectively. In this paper, particle swarm optimization(PSO) algorithm and genetic algorithm(GA) are selected.

\subsection{Steps and parameters of the optimal charge simulating method}

The program flow chart of the optimized electric field inverse operation algorithm is shown in Figure 3.

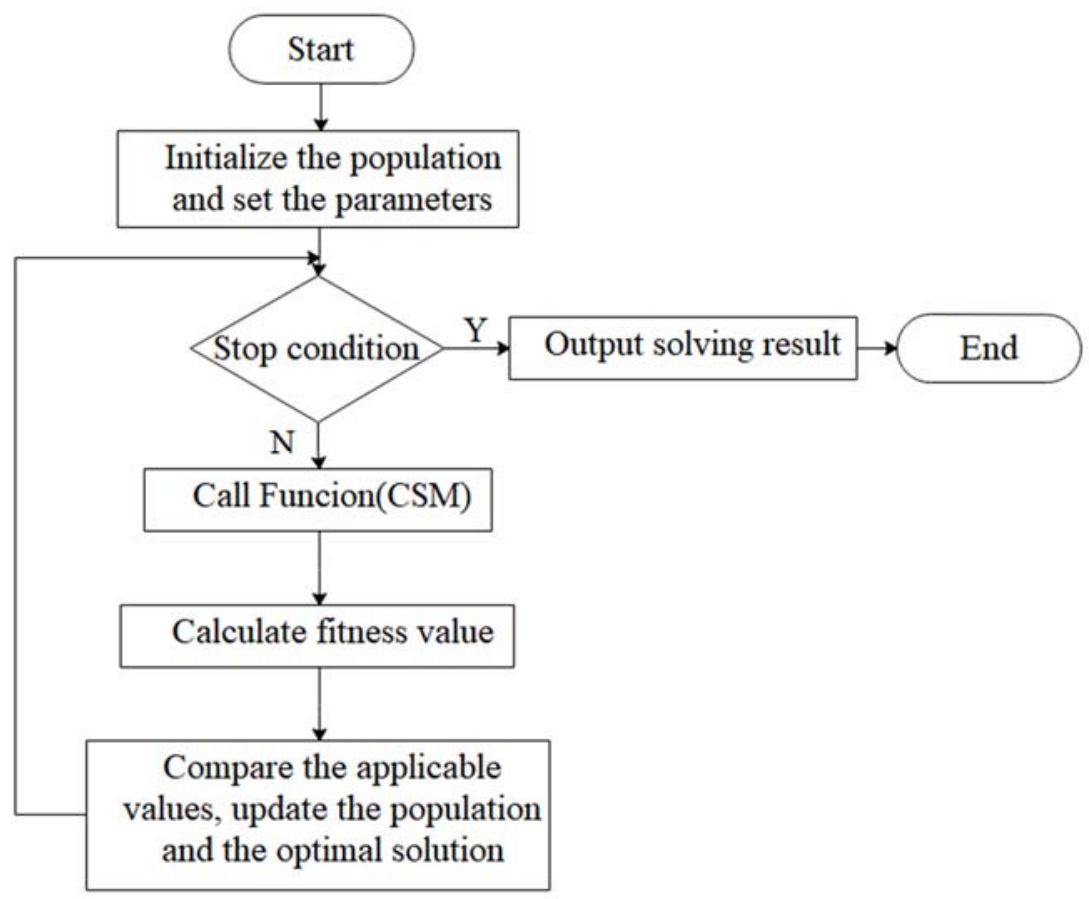

Fig. 3. The flow chart of optimization electric field inverse operation algorithm.

For the algorithm theory of the two intelligent algorithms, it is necessary to set the initial population number, strategy parameters, update population parameters and stop conditions. The above parameters and conditions in the algorithm are shown in the following table. 
Table 1. The algorithm parameter of intelligent algorithm.

\begin{tabular}{|l|l|}
\hline Intelligence Algorithm & Algorithm parameter \\
\hline \multirow{3}{*}{ PSO } & $\begin{array}{l}\text { Number of Iterations }=100, \text { Maximum Speed of Change }=20 \%, \\
\text { Population Size }=20\end{array}$ \\
\cline { 2 - 2 } & Acceleration Coefficient $c_{1}=c_{2}=2$, Inertia Weight $\omega=0.8$ \\
\hline \multirow{2}{*}{ GA } & Number of Iterations $=100$, Population Size $=20$ \\
\cline { 2 - 2 } & Variation Probability $=1 \%$, Cross Probability $=0.9$ \\
\hline
\end{tabular}

\subsection{Fitness function}

The adaptive values of individuals are calculated for comparison in the intelligent algorithm, which is used to update the population and select the optimal solution. Therefore, the appropriate fitness function is needed to be constructed.

Since potential value is composed of voltage value and phase value, and there are $n$ potential values, fitness value can be written as:

$$
f f_{v}=\frac{1}{n}\left(\sum_{i=1}^{n}\left|\frac{\bar{V}_{t e s t, i}-\bar{V}_{i}}{\bar{V}_{i}}\right|+\sum_{i=1}^{n}\left|\frac{V_{t e s t, i}^{*}-V_{i}^{*}}{V_{i}^{*}}\right|\right)
$$

Where $n$ is the phase number of transmission lines, $\bar{V}_{\text {test }, i}$ and $V_{\text {test }, i}^{*}$ are the calculated voltage value and phase value of the $i$-phase transmission line respectively, $\bar{V}_{i}$ and $V_{i}^{*}$ are the given voltage value and given phase value of the $i$-phase transmission line respectively. The solution results of the voltage and phase values are obtained by calling Function(CSM), and then the fitness value is calculated. The smaller the difference between the calculated value and the known value, that is, the smaller the fitness value $f f_{v}$, the better the result.

\section{Experimental results and analysis of optimal charge simulating method}

The calculation example in this chapter takes the single-loop horizontal 220kV high-voltage transmission line as an example. The three-phase transmission lines A, B and C are horizontally arranged, and the radius of splitting conductor is $0.4 \mathrm{~m}$. The tower parameters are shown in Figure 4-1.

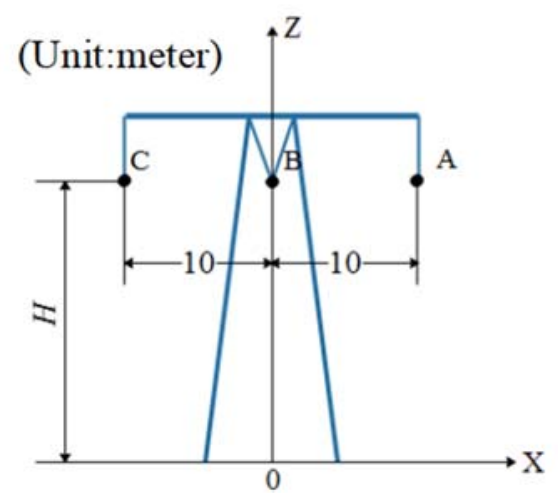

Fig. 4. Schematic diagram of a high voltage transmission tower 
Meanwhile, the transmission line and its surrounding environment are simplified. The transmission line is regarded as a infinite length straight line that parallel to the ground, the split wire is simplified as an equivalent wire, and the ground is an insulating surface with zero potential.

The electric field value is calculated under this example. The calculation results of electric field intensity distribution at a height 5 meters above the ground are shown in Fig. 5 . Take the electric field intensity value at the appropriate position from the calculation results. In this experiment, the measuring points arranged horizontally with a height of $5 \mathrm{~m}$ are selected, which corresponding to the position of three-phase transmission lines A, B and C on X-axis.

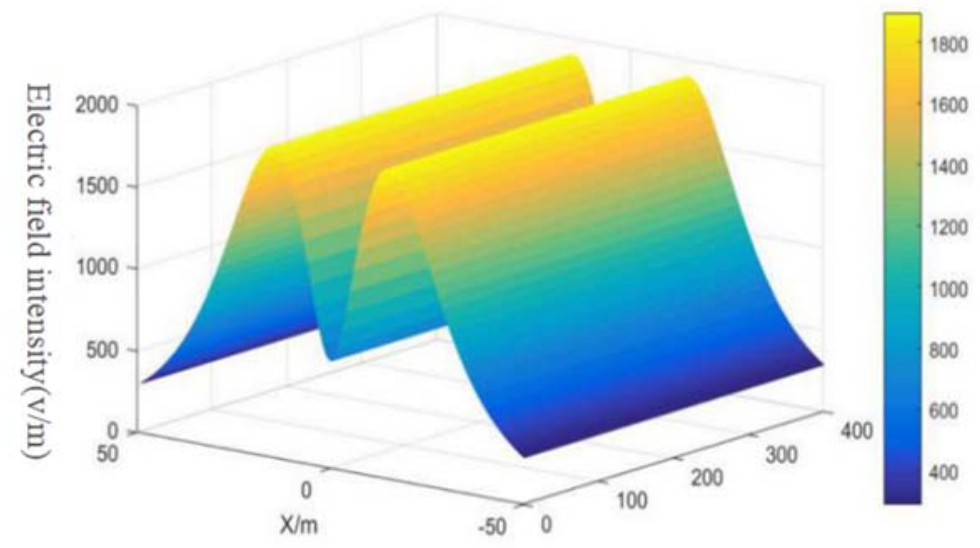

Fig. 5. Schematic diagram of electric field intensity.

The OCSM-PSO and the OCSM-GA all set the number of iterations as 100 , and the population size as 20 . The value range of population is $100 \sim 500$, corresponding to the height range of wire is set as $100 \mathrm{dm} \sim 500 \mathrm{dm}$. The number of iterations is set as the stop condition of the algorithm. Finally, the optimal individual in the population obtained has the best fitness value, which is the optimal solution of conductor height.

The program of OCSM is run on the software Matlab 2016b. The CPU configuration of the computer is $\mathrm{I} 5-8265 \mathrm{U}$, and the RAM is $8 \mathrm{~GB}$. The optimal results, fitness value and calculation time of the OCSM-PSO and the OCSM-GA shown in Figure 6 and Table 2.

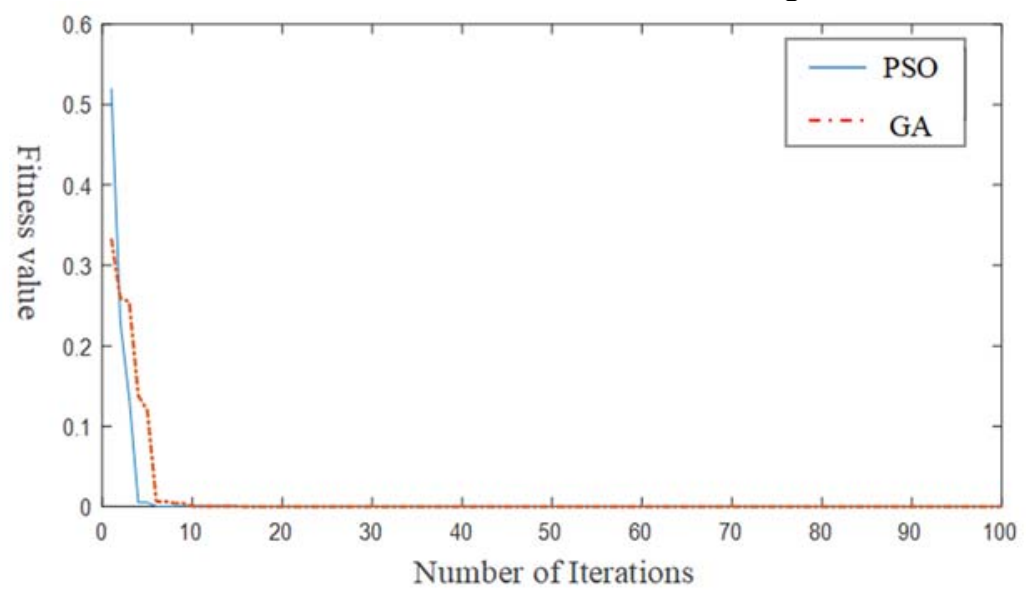

Fig. 6. Schematic diagram of iterative calculation. 
Table 2. Calculation results of OCSM

\begin{tabular}{|l|l|l|}
\hline Parameter of OCSM & OCSM-PSO & OCSM-GA \\
\hline The Number of Iterations to Obtain Stable Results & 25 & 33 \\
\hline Solution Result of Conductor Height $/(\mathrm{m})$ & 25.0 & 25.0 \\
\hline Reference Voltage $/(\mathrm{V})$ & 133367.91 & 133367.91 \\
\hline Reference Phase & $45^{\circ}, 165^{\circ}, 285^{\circ}$ & $45^{\circ}, 165^{\circ}, 285^{\circ}$ \\
\hline Fitness Values & $12.0366 \times 10^{-6}$ & $13.4125 \times 10^{-6}$ \\
\hline Calculation Time/(s) & 5.3248 & 5.9705 \\
\hline
\end{tabular}

It can be clearly seen from the above figure and table that the OCSM-PSO iterates for 25 times to obtain the stable calculation result and solve the single optimal solution in the optimal population. That is, the wire height is 25.0 meters, which is in line with the wire height set in the calculation example, and the best fitness value is $12.0366 \times 10^{-6}$. Through of 33 iterative computations of the OCSM-GA, the stable calculation results were obtained. The solution result is 25.0 meters, and the best fitness value is $13.4125 \times 10^{-6}$. Both intelligent algorithms obtain accurate results, and the fitness value of $10^{-6}$ power is obtained to ensure the accuracy of the results. Compared with the two algorithms, the computation time of the OCSM-PSO is slightly less than that of the OCSM-GA, with higher computational efficiency and accuracy.

\section{Conclusion}

In this paper, the intelligent algorithm with global optimization and the CSM are introduced to solve the problem of inverse calculation. Two different intelligent algorithms, particle swarm optimization algorithm and genetic algorithm are combined with CSM. The same optimal solution and the similar best fitness value are solved, which prove the general effectiveness and solving accuracy of using intelligent algorithm for the OCSM. And The computational efficiency of the two algorithms is compared.

The focus of this paper is to describe a method to optimize the inverse operation of electric field, and provide a method to solve the inverse operation problem when there are multiple unknowns, which has a guiding significance for the engineering application of nondestructive determination through electric field inverse operation. In addition, considering the actual situation of the transmission line, it is necessary to further study the three-dimensional model and method of electric field calculation, and solve the matrix problems by combining regularization, so as to improve the ill condition and instability that may occur in the inverse operation.

\section{Acknowledgements}

Science and Technology Project of State Grid Sichuan Electric Power Company (5219621900BG).

\section{References}

1. B. Chen, L. Du, K. Liu et al.: Measurement error estimation for capacitive voltage transformer by insulation parameters [J]. Energies, 3, 1-14(2017).

2. Shao F.: Operation Maintenance and Overhauling Technology of UHV Transmission Line [M]. China Electric Power Press, Beijing(2016). 
3. Feng Y., Wang X., Chen X. et al.: Influence of Circuit Parameters of Capacitance Voltage Transformer on Power Grid Harmonic Voltage Measurement [J]. Proceedings of the CSEE, 28, 4968-4975(2014).

4. Naoto M., Satoshi I.: Development of a modified envelope correlation method based on maximum-likelihood method and application to detecting and locating deep tectonic tremors in western Japan [J]. Earth, Planets \& Space, 1, 1-14(2019).

5. Ahmadi L., Hosseini S. M. and Shishegar A. A.: Solving Inverse Electromagnetic Problems Using Deep Learning [C]. 2020 28th Iranian Conference on Electrical Engineering(ICEE), 1-4(2020).

6. Si D., Wang J., Wei G. et al.: Method and Experimental Study of Voltage Measurement Based on Electric Field Integral With Gauss-Legendre Algorithm[J]. IEEE Transactions on Instrumentation and Measurement, 69, 2771-2778(2020).

7. Qin G.: Research on Radio Interference and Human Electro-induction under Parallel AC Transmission Line with Corridor [D]. Chongqing University(2016).

8. Zhang Z., Lei J., Xie X., et al. Optimization algorithm of inverse problem for a power-frequency electric field [J], International Transactions on Electrical Energy Systems, 1, 89-98(2015).

9. Xiao D., Xie Y., Ma Q., et al.: Non-contact voltage measurement of threephase overhead transmission line based on electric field inverse calculation [J], IET Generation, Transmission \& Distribution, 12, 2952-2957(2018).

10. Yang F.: Forward and Inverse Problems of Power Frequency Electric Field of Power Transmission and Transformation Equipment and Research on Electromagnetic Environment [D]. Chongqing University(2008).

11. Xiao D., Jiang K., Liu H., et al.: Inversion and Inversion Method from Power Frequency Magnetic Field Data to Three-phase Current of Overhead Transmission Lines[J]. Proceedings of the CSEE, 5, 1438-1444(2016).

12. An S., Yang S., Li T.: Improved Tabu Search Algorithm and Its Application in Inverse Problems of Electromagnetic Field [J]. Power System Protection and Control, 24, 30-33(2010).

13. Wang R., Tian J., Wu F., et al.: Optimisation electric field inverse solving algorithm for non-destructive detection of voltage on transmission lines [J]. IET Generation, Transmission \& Distribution, 13, 5423-5430(2019). 